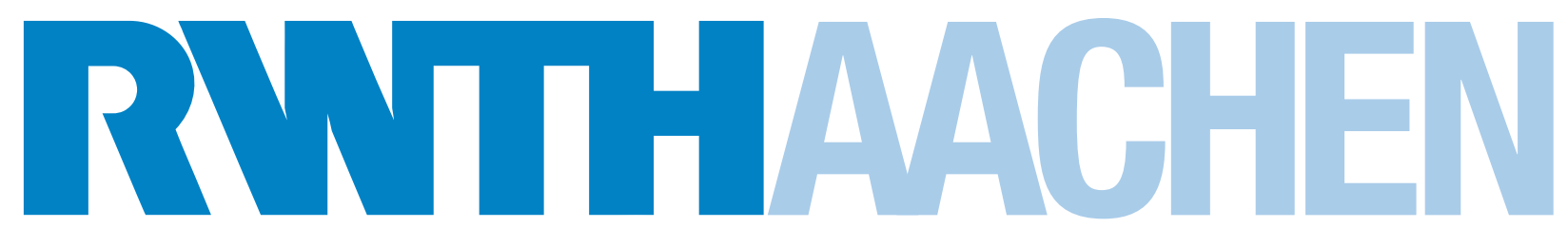

RHEINISCH-WESTFÄLISCHE TECHNISCHE HOCHSCHULE AACHEN Institut für Mathematik

\author{
A characterisation of \\ inner product spaces \\ by the maximal circumradius of spheres \\ by
}

Sebastian Scholtes

Report No. 53

2012

February

2012

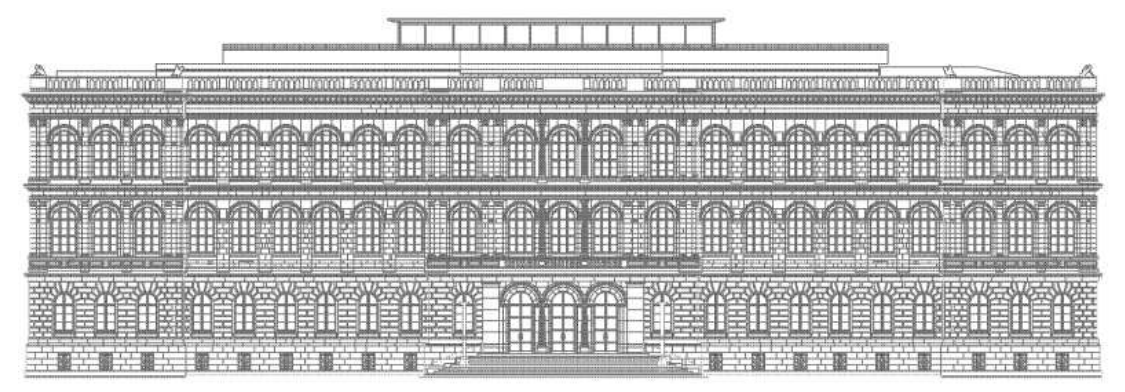

Institute for Mathematics, RWTH Aachen University

Templergraben 55, D-52062 Aachen

Germany 


\title{
A characterisation of inner product spaces by the maximal circumradius of spheres
}

\author{
Sebastian Scholtes
}

February 2, 2012

\begin{abstract}
We give a new characterisation of inner product spaces amongst normed vector spaces in terms of the maximal cirumradius of spheres. It turns out that a normed vector space $(X,\|\cdot\|)$ with $\operatorname{dim} X \geq 2$ is an inner product space if and only if all spheres are not degenerate, i.e. the maximal circumradius of points on the sphere equals the radius of the sphere or more formally
\end{abstract}

$$
\sup _{\substack{u, v, w \in \partial B_{r}\left(x_{0}\right) \\ u \neq v \neq w \neq u}} r(u, v, w)=r \quad \text { for all } x_{0} \in X \text { and all } r>0 .
$$

Mathematics Subject Classification (2000): 46C15; 46B20

It is an important and particularly well researched topic to find conditions that classify inner product spaces amongst, say, mere normed vector spaces. This area of research really took off in 1935, where several of these characterisations were given. Best-know of these characterisations is the one by Jordan and von Neumann [JvN35]. They showed that an inner product, which induces the norm, can be defined in a normed vector space if and only if the parallelogram law holds. For detailed historical information we refer the reader to the introduction and chronological publication list in [Ami86]. A good overview of the criteria available in their respective times can be found in [Day62, Ist87] and, of course, the very comprehensive [Ami86]. The book [AST10] gives a survey of the many characterisations in terms of norm derivatives. Up to the present day this topic still remains very active and fruitful, so that, because of the sheer number of publications in this area, we restrain ourselves from trying to give a survey and instead refer the interested reader to the books mentioned above and the papers which are cited below as a starting point.

In what follows we give a, to the best of our knowledge, new characterisation of inner product spaces amongst normed vector spaces, which involves the maximal circumradius of points in spheres. In a metric space $(X, d)$ the circumradius $r(u, v, w)$ of three mutually distinct points $u, v, w \in X$ is defined to be the circumradius of the triangle constituted by the isometric embedding of these three points in the Euclidean plane and can be written as

$$
r(u, v, w)=\frac{a b c}{\sqrt{(a+b+c)(a+b-c)(a-b+c)(-a+b+c)}}=\frac{a b c}{\sqrt{-D(u, v, w)}}
$$


where $a:=d(u, v), b:=d(v, w), c:=d(w, u)$ and incidentally $D(u, v, w)$ is the CayleyMenger determinant, see [Blu70, 40, pp. 97-99, especially Ex. 3]. If $D(u, v, w)=0$ we set $r(u, v, w)=\infty$. This quantity plays an important role in geometric curvature energies and ideal knots, where various curvature energies are defined in terms of $r(u, v, w)$. The most established of these energies are probably the thickness $\Delta$ and the integral p-Menger curvature $\mathcal{M}_{p}$ of a metric space $(X, d)$, defined by

$$
\Delta[X]:=\inf _{\substack{u, v, w \in X \\ u \neq v \neq w \neq u}} r(u, v, w), \quad \mathcal{M}_{p}(X):=\int_{X} \int_{X} \int_{X} \frac{1}{r(u, v, w)^{p}} \mathrm{~d} \mathcal{H}^{\alpha}(u) \mathrm{d} \mathcal{H}^{\alpha}(v) \mathrm{d} \mathcal{H}^{\alpha}(w) .
$$

Concerning these curvature energies we want to refer the reader to [GM99] for the thickness, and [Hah08, SSvdM10, Sch12] and the references therein for the integral Menger curvature and "intermediate energies". The notions above were also the cause why the author "accidentally" found the present characterisation of inner product spaces. We want to mention the role of integral Menger curvature for $p=2$ in the solution of the Painlevé problem, i.e. to find geometric characterisations of removable sets for bounded analytic functions, see [Paj02] for a detailed presentation and references.

In [Kle60] a characterisation of inner product spaces under normed vector spaces in terms of the $X$-radius

$$
r_{X}(M):=\inf \left\{r \in(0, \infty) \mid \exists x_{0} \in X: M \subset \bar{B}_{r}\left(x_{0}\right)\right\}
$$

of a bounded subset $M$ of a normed vector space $X$ were given. If $\operatorname{dim} X \geq 3$ the existence of an inner product is equivalent to $r_{C}(C)=r_{X}(C)$ for every bounded convex set $C \subset X$. Further results connected with the classic circumradius of points can be found in [AGT96], where a characterisation of inner product spaces is given in terms of the radius of the inscribed circle and the circumradius of triangles being comprised by two independent vectors. More precisely they use two notions of each radius: one notion is defined only in terms of distances and the other additionally involves an inner product. Both these notions agree in inner product spaces. To make sense of the second notion for normed vector spaces, a generalized inner product on normed vector spaces, namely the norm derivative, is used. Now it can be shown that inner product spaces are exactly those normed vector spaces in which these two notions of radius agree. In case of the inscribed circle this was restricted to spaces of dimension larger than two and for the circumradius to spaces of dimension at least three with the additional restriction of being strictly convex. Later on in [Tom05] one of these authors showed that a strictly convex normed vector space of dimension larger than three is an inner product space if and only if a circumcenter, in the sense of the intersection of the perpendicular bisectors of the edges, exists for certain types of triangles. In general there may be more than one point in this intersection, i.e. more than one circumcenter. Subsequently it was shown that a strictly convex normed vector space is an inner product space, if and only if for every collection of points on a sphere centred at the origin one of these circumcenters is the origin.

The paper is organized as follows: After looking into the behaviour of points not fulfilling the parallelogram law we shortly prove a well-known characterisation of inner product spaces by Wilson involving the Euclidean four point property. We then briefly remark on a space of four points not embeddable in inner product spaces and aspects of geometry 
in $\left(\mathbb{R}^{2},\|\cdot\|_{\infty}\right)$ in general. After that we give the characterisation of inner product spaces announced in the title.

\section{Acknowledgement}

The author whishes to thank his advisor Heiko von der Mosel for constant support and encouragement.

\section{A characterisation of inner product spaces in terms of the maximal circumradius of spheres}

Definition 1.1 (Inner product space, non inner product space).

Let $(X,\|\cdot\|)$ be a normed real vector space. We call $(X,\|\cdot\|)$ an inner product space, or short ips, if there exists an inner product $\langle\cdot, \cdot\rangle$ on $X$, which induces the norm, i.e. $\|x\|=\sqrt{\langle x, x\rangle}$ for all $x \in X$. If there exists no such inner product on $X$, we call $(X,\|\cdot\|)$ a non inner product space, or short nips. We refer to a sphere $\partial B_{r}\left(x_{0}\right)$ as being degenerate if there are points $u, v, w \in \partial B_{r}\left(x_{0}\right)$, such that $r(u, v, w)>r$.

We start by investigating what characterises points for which the parallelogram law does not hold.

Lemma 1.2 (Properties of points not fulfilling the parallelogram law).

Let $\left(X,\|\cdot\|_{X}\right)$ be a normed vector space and $u, v \in X$ points which do not satisfy the parallelogram law, i.e.

$$
2\|u\|_{X}^{2}+2\|v\|_{X}^{2} \neq\|u+v\|_{X}^{2}+\|u-v\|_{X}^{2} .
$$

Then $\left(\{0, u, v,-v\}, d_{X}\right)$, where $d_{X}$ is the metric $d_{X}(a, b)=\|a-b\|_{X}$, is not isometrically embeddable in any inner product space.

Proof. Step 1 From (1) we know that $u \notin\{0, v,-v\}$ and $v \notin\{0, u,-u\}$. Assume that there exists an isometric embedding $\varphi:\left(\{0, u, v,-v\}, d_{X}\right) \rightarrow\left(V,\|\cdot\|_{V}\right)$ into an inner product space. Without loss of generality, by translating by $-\varphi(0)$ if necessary, we can assume $\varphi(0)=0$. We now have

$$
\|\varphi(u)\|_{V}=\|\varphi(u)-\varphi(0)\|_{V}=d_{V}(\varphi(u), \varphi(0))=d_{X}(u, 0)=\|u-0\|_{X}=\|u\|_{X}
$$

and by the same argument $\|\varphi(v)\|_{V}=\|v\|_{X}=\|-v\|_{X}=\|\varphi(-v)\|_{V}$. The parallelogram law for $v$ and $-v$ gives us

$$
\begin{aligned}
4\|v\|_{X}^{2} & =2\|v\|_{X}^{2}+2\|v\|_{X}^{2}=2\|\varphi(v)\|_{V}^{2}+2\|\varphi(-v)\|_{V}^{2} \\
& =\|\varphi(v)+\varphi(-v)\|_{V}^{2}+\|\varphi(v)-\varphi(-v)\|_{V}^{2} \\
& =\|\varphi(v)+\varphi(-v)\|_{V}^{2}+d_{V}(\varphi(v), \varphi(-v))^{2} \\
& =\|\varphi(v)+\varphi(-v)\|_{V}^{2}+d_{X}(v,-v)^{2} \\
& =\|\varphi(v)+\varphi(-v)\|_{V}^{2}+\|v-(-v)\|_{X}^{2} \\
& =\|\varphi(v)+\varphi(-v)\|_{V}^{2}+4\|v\|_{X}^{2},
\end{aligned}
$$


so that $\|\varphi(v)+\varphi(-v)\|_{V}=0$ and consequently $\varphi(-v)=-\varphi(v)$.

Step 2 This gives us

$$
\begin{aligned}
& 2\|u\|_{X}^{2}+2\|v\|_{X}^{2}=2\|\varphi(u)\|_{V}^{2}+2\|\varphi(v)\|_{V}^{2} \\
& \quad=\|\varphi(u)+\varphi(v)\|_{V}^{2}+\|\varphi(u)-\varphi(v)\|_{V}^{2} \\
& \quad=\|\varphi(u)-\varphi(-v)\|_{V}^{2}+\|\varphi(u)-\varphi(v)\|_{V}^{2} \\
& \quad=\|u-(-v)\|_{X}^{2}+\|u-v\|_{X}^{2}
\end{aligned}
$$

which contradicts $(1)$.

We now have the means to prove en passant the most interesting implication, here (iv) implies (i), in a variation of a well-known theorem of Wilson [Wil32, 12. Theorem], for the special case of normed vector spaces.

Proposition 1.3 (Characterisation of ips via Euclidean four point property). Let $\left(X,\|\cdot\|_{X}\right)$ be a normed vector space. Then the following are equivalent

(i) $\left(X,\|\cdot\|_{X}\right)$ is an inner product space,

(ii) there exists an inner product space $\left(V,\|\cdot\|_{V}\right)$ such that $\left(X,\|\cdot\|_{X}\right)$ is isometrically embeddable in $\left(V,\|\cdot\|_{V}\right)$,

(iii) all sets $F=\{u, v, w, x\} \subset X$ are isometrically embeddable in $\left(\mathbb{R}^{3},\|\cdot\|_{2}\right)$,

(iv) for all sets $F=\{u, v, w, x\} \subset X$ exists an inner product space $\left(V(F),\|\cdot\|_{V(F)}\right)$ in which $F$ is isometrically embeddable.

Proof. Clearly (i) implies (ii) and (iii) implies (iv). Now for (ii) implies (iii). By (ii) a given set $\{u, v, w, x\} \subset X$ is isometrically mapped to four points in the inner product space $V$ and therefore also to four points in the completion of this inner product space, which again is an inner product space. This means (iii) is simply a consequence of the observation that the Euclidean four-point property of [Wil32], see also [Day62, (e4pp-0), p.116], holds for inner product spaces. The remaining direction is the contrapositive of Lemma 1.2, since a normed vector space is an inner product space if and only if the parallelogram law holds for all $u, v \in X$.

It is kind of surprising that although any three points in a pseudometric space can be isometrically embedded in the plane, there are four points in a Banach space, in the example we will present even the center and three points on the boundary of a ball, which cannot be isometrically embedded in any inner product space. A similar space and the nonexistence of isometric embeddings in Euclidean spaces can be found in [Rob06]. We refer the reader to [YZ84] for more complicated examples and conditions on sets which cannot be isometrically embedded in any Hilbert space.

\section{Example 1.4 (Four points not isometrically embeddable in ips).}

Consider the Banach space $\left(\mathbb{R}^{2},\|\cdot\|_{\infty}\right)$ and

$$
\theta:=(0,0), u:=(0,1), v:=(1,0),-v=(-1,0) \in \mathbb{R}^{2} .
$$

Then according to Lemma 1.5 for any inner product space $(V,\langle\cdot, \cdot\rangle)$ there exists no, possibly nonlinear, isometry $\varphi:\left(\{\theta, u, v,-v\},\|\cdot\|_{\infty}\right) \rightarrow(V,\langle\cdot, \cdot\rangle)$, since one can easily check that $r(u, v,-v)=\infty$. Alternatively one can verify that the parallelogram law does not hold for $u$ and $v$ and then use Lemma 1.2. 
In Lemma A.3 in the appendix we continue this example and show that we find points of arbitrary circumradius on any sphere in $\left(\mathbb{R}^{2},\|\cdot\|_{\infty}\right)$.

\section{Lemma 1.5 (Embedding of three points on a sphere and their center).}

Let $(X, d)$ be a metric space and $x_{0}, u, v, w \in X$ mutually distinct points, such that $d\left(x_{0}, x\right)=r$ and $r>0$ for all $x \in\{u, v, w\}$. Then $\left\{x_{0}, u, v, w\right\}$ is isometrically embeddable in 3-dimensional Euclidean space - which incidentally is the same as being isometrically embeddable in any inner product space - if and only if $r(u, v, w) \leq r$.

Proof. Let $r(u, v, w)<\infty$, or else see Case 3. Without loss of generality we start by embedding the three points $\{u, v, w\}$ isometrically in the $x y$ plane in $\mathbb{R}^{3}$ with a mapping $\varphi$, such that the the center of the circumcircle of $\varphi(x), \varphi(y), \varphi(z)$ is the origin; and have to investigate wether we can extend $\varphi$ isometrically to $x_{0}$, i.e. $\varphi(u), \varphi(v), \varphi(w) \in \partial B_{r}\left(\varphi\left(x_{0}\right)\right)$. Case 1 If $r(u, v, w) \leq r$ we can place $\varphi\left(x_{0}\right)=\left(0,0, \sqrt{r^{2}-r(u, v, w)^{2}}\right)$ in the appropriate distance along the $z$-axis.

Case 2 If $r<r(u, v, w)$ the distance $r$, where $\varphi\left(x_{0}\right)$ would have to be placed, if we expect our embedding to be isometrically, is too short to reach the $z$-axis.

Case 3 If $r(u, v, w)=\infty$ the mutually distinct points $\varphi(u), \varphi(v), \varphi(w)$ lie on a straight line. In Euclidean space a line can meet a sphere at most two times, see Lemma A.1, such that there is no $a \in \mathbb{R}^{3}$ with $\varphi(u), \varphi(v), \varphi(w) \in \partial B_{r}(a)$.

Lemma 1.6 (All spheres in non inner product spaces are degenerate).

Let $(X,\|\cdot\|)$ be a normed vector space, which is a non inner product space. Then for all $x_{0} \in X$ and all $r>0$ there exist mutually distinct $u, v \in \partial B_{r}\left(x_{0}\right)$ such that $r(u, v,-v)>r$.

Proof. Without loss of generality we can assume $x_{0}=0$, because we can always translate by $-x_{0}$. By scaling the two vectors in the parallelogram law with $r$, we know from [Day47, Theorem 2.1] that for each $r>0$ exist vectors $u, v \in \partial B_{r}(0)$, such that

$$
\|u+v\|^{2}+\|u-v\|^{2} \neq 2\|u\|^{2}+2\|v\|^{2}=4 r^{2}
$$

so that by Lemma 1.2 the points $\{0, u, v,-v\}$ are not isometrically embeddable in any inner product space. Considering Lemma 1.5 we have $r<r(u, v,-v)$.

Lemma 1.7 (Supremum is attained).

Let $(V,\|\cdot\|)$ be an inner product space, $\operatorname{dim} V=2$. Then for $x_{0} \in V, r>0$ and all pairwise distinct $u, v, w \in \partial B_{r}\left(x_{0}\right)$ we have $r(u, v, w)=r$.

Proof. Since $\partial B_{r}\left(x_{0}\right)=x_{0}+\partial B_{r}(0)$ and $r(u, v, w)=r\left(u+x_{0}, v+x_{0}, w+x_{0}\right)$ we can safely assume $x_{0}=0$. Let $P \subset V$ be a two-dimensional subspace. Then $P$ is a two-dimensional inner product space, which is isometrically isomorphic to the Euclidean plane $\left(\mathbb{R}^{2},\|\cdot\|_{2}\right)$, as can easily been seen by choosing an orthonormal base $\left(b_{1}, b_{2}\right)$ of $P$ and noting that $\left\|\alpha b_{1}+\beta b_{2}\right\|=\sqrt{\alpha^{2}+\beta^{2}}=\|(\alpha, \beta)\|_{2}$, which gives rise to an isometric isomorphism $\varphi$. Since the circumradius only depends on the distances of points we have $r(x, y, z)=$ $r(\varphi(x), \varphi(y), \varphi(z))=r$.

We should remark that the condition $\operatorname{dim} X \geq 2$, we will impose on our space, is not really a restriction, as every one-dimensional space is an inner product space, because the norm is essentially a positive constant times the absolute value. 
Definition 1.8 (The quantity $S(M)$ ).

To shorten notation we denote

$$
S(M):=\sup _{\substack{u, v, w \in M \\ u \neq v \neq w \neq u}} r(u, v, w) \in(0, \infty]
$$

for a subset $M$ of a metric space $(X, d)$.

Theorem 1.9 (Characterisation of ips via circumradius).

Let $(X,\|\cdot\|)$ be a normed vector space, $\operatorname{dim} X \geq 2$. The following items are equivalent

(i) $(X,\|\cdot\|)$ is an inner product space,

(ii) for all $x_{0} \in X$ and all $r>0$ we have $S\left(\partial B_{r}\left(x_{0}\right)\right)=r$,

(iii) there exists a $x_{0} \in X$ and a $r>0$, such that $S\left(\partial B_{r}\left(x_{0}\right)\right)=r$.

Proof. We start by proving (i) $\Rightarrow($ ii). If $(X,\|\cdot\|)$ is an inner product space and we take $x_{0}, r, u, v, w$ as in (ii) then $S\left(\partial B_{r}\left(x_{0}\right)\right) \leq r$ by Lemma 1.5 and "=" by Lemma 1.7. The implication (ii) $\Rightarrow$ (iii) is clearly true. Now assume (iii), i.e. there exists a sphere, which is not degenerate. Then by the contrapositive of Lemma 1.6 this implies (i).

Taking the risk of repeating ourselves, we want to spell out, how one could go about in finding out if a normed space is an inner product space.

Corollary 1.10 (Computing a single $S\left(\partial B_{r}\left(x_{0}\right)\right)$ decides about ips or nips). Let $(X,\|\cdot\|)$ be a normed vector space, $\operatorname{dim} X \geq 2$ and $x_{0} \in X, r>0$. Then $S\left(\partial B_{r}\left(x_{0}\right)\right) \geq$ $r$ and

- if $r=S\left(\partial B_{r}\left(x_{0}\right)\right)$ then $(X,\|\cdot\|)$ is an inner product space,

- if $r<S\left(\partial B_{r}\left(x_{0}\right)\right)$ then $(X,\|\cdot\|)$ is a non inner product space.

Proof. Direct consequence of Theorem 1.9.

\section{A Appendix}

Lemma A.1 (Intersection of sphere and straight line in inner product spaces). In an inner product space $(V,\|\cdot\|)$ a sphere and a straight line can coincide in at most two points.

Proof. Let $x_{0}, a, v \in V,\|v\|=1, r>0$. Consider the sphere $S:=\partial B_{r}\left(x_{0}\right)$ and the straight line $L:=a+\mathbb{R} v$. We have $y \in S \cap L$ if $y=a+t v$ for $t \in \mathbb{R}$ and $\left\|a+t v-x_{0}\right\|=r$, which gives us

$$
\left\|a+t v-x_{0}\right\|^{2}=\left\langle\left(a-x_{0}\right)+t v,\left(a-x_{0}\right)+t v\right\rangle=\left\|a-x_{0}\right\|^{2}+2\left\langle a-x_{0}, v\right\rangle t+t^{2} .
$$

This is a quadratic polynomial, which has at most two solutions.

Remark A.2 (In nips lines and spheres can intersect in many points).

Considering the shape of $\partial B_{1}(0)$ in $\left(\mathbb{R}^{2},\|\cdot\|_{\infty}\right)$ we see that the conclusion of Lemma A.1 does not hold if we drop the assumption, that $(V,\|\cdot\|)$ has a norm induced by an inner product. 
Lemma A.3 (Circumradius of points on spheres in $\left(\mathbb{R}^{2},\|\cdot\|_{\infty}\right)$ ).

We consider the Banach space $\left(\mathbb{R}^{2},\|\cdot\|_{\infty}\right)$. Let $x_{0} \in \mathbb{R}^{2}$ and $\rho>0$. For all $d \in(0, \infty]$ exist $u, v, w \in \partial B_{\rho}\left(x_{0}\right)$, such that $r(u, v, w)=d$.

Proof. Without loss of generality we can assume $x_{0}=0$. By scaling, i.e. $r(c u, c v, c w)=$ $\operatorname{cr}(u, v, w)$ for $c>0$, we only have to verify our claim for, say, $\rho=1$.

Step 1 Choose $u=(-1,1-s), v=(-1+s, 1)$ and $w=(-1,1)$ for $s \in(0,2)$. If we embed $u, v, w$ isometrically in the Euclidean plane we get an equilateral triangle with side lengths $s$, whose circumradius is given by

$$
r(u, v, w)=\frac{s^{3}}{\sqrt{3 s^{4}}}=\frac{s}{\sqrt{3}}
$$

so that we proved the proposition for $d \in(0,2 / \sqrt{3})$.

Step 2 Choose $u=(-1,1-s), v=(1,1-s)$ and $w=(0,1)$ for $s \in(1,2]$. By isometrically embedding these points in the Euclidean plane we get an isosceles triangle with side lengths $s, s$ and 2 , such that

$$
r(u, v, w)=\frac{2 s^{2}}{\sqrt{(2+2 s) \cdot 2 \cdot 2 \cdot(-2+2 s)}}=\frac{s^{2}}{2 \sqrt{s^{2}-1}},
$$

which proves the proposition for $[2 / \sqrt{3}, \infty)$. The remaining case $r(u, v, w)=\infty$ was already treated in Example 1.4.

\section{References}

[AGT96] C. Alsina, P. Guijarro, and M. S. Tomás, Characterizations of inner product structures involving the radius of the inscribed or circumscribed circumference, Arch. Math. (Brno) 32 (1996), no. 3, 233-239.

[Ami86] Dan Amir, Characterizations of inner product spaces, Operator Theory: Advances and Applications, vol. 20, Birkhäuser Verlag, Basel, 1986.

[AST10] Claudi Alsina, Justyna Sikorska, and M. Santos Tomás, Norm derivatives and characterizations of inner product spaces, World Scientific Publishing Co. Pte. Ltd., Hackensack, NJ, 2010.

[Blu70] Leonard M. Blumenthal, Theory and applications of distance geometry, Second edition, Chelsea Publishing Co., New York, 1970.

[Day47] Mahlon M. Day, Some characterizations of inner-product spaces, Trans. Amer. Math. Soc. 62 (1947), 320-337.

[Day62] _ _ Normed linear spaces, second ed., Springer-Verlag, New York, 1962.

[GM99] Oscar Gonzalez and John H. Maddocks, Global curvature, thickness, and the ideal shapes of knots, Proc. Natl. Acad. Sci. USA 96 (1999), no. 9, 4769-4773 (electronic).

[Hah08] Immo Hahlomaa, Menger curvature and rectifiability in metric spaces, Adv. Math. 219 (2008), no. 6, 1894-1915. 
[Ist87] Vasile Ion Istrăţescu, Inner product structures, Mathematics and its Applications, vol. 25, D. Reidel Publishing Co., Dordrecht, 1987.

[JvN35] P. Jordan and J. von Neumann, On inner products in linear, metric spaces, Ann. of Math. (2) 36 (1935), no. 3, 719-723.

[Kle60] Victor Klee, Circumspheres and inner products, Math. Scand. 8 (1960), 363370 .

[Paj02] Hervé Pajot, Analytic capacity, rectifiability, menger curvature and the cauchy integral, Lecture Notes in Mathematics, vol. 1799, Springer-Verlag, Berlin, 2002 .

[Rob06] P. L. Robinson, The sphere is not flat, Amer. Math. Monthly 113 (2006), no. $2,171-173$.

[Sch12] Sebastian Scholtes, Tangency properties of sets with finite geometric curvature energy, Preprint 52, RWTH Aachen University, Institut f. Mathematik, 2012.

[SSvdM10] Paweł Strzelecki, Marta Szumańska, and Heiko von der Mosel, Regularizing and self-avoidance effects of integral Menger curvature, Ann. Scuola Norm. Sup. Pisa Cl. Sci. (5) Vol. IX (2010), 1-43.

[Tom05] M. S. Tomás, Circumcenters in real normed spaces, Boll. Unione Mat. Ital. Sez. B Artic. Ric. Mat. (8) 8 (2005), no. 2, 421-430.

[Wil32] W. A. Wilson, A Relation Between Metric and Euclidean Spaces, Amer. J. Math. 54 (1932), no. 3, 505-517.

[YZ84] Lu Yang and Jing Zhong Zhang, Metric spaces which cannot be isometrically embedded in Hilbert space, Bull. Austral. Math. Soc. 30 (1984), no. 2, 161-167.

Sebastian Scholtes

Institut für Mathematik

RWTH Aachen University

Templergraben 55

D-52062 Aachen, Germany

sebastian.scholtes@rwth-aachen.de 


\section{Reports des Instituts für Mathematik der RWTH-Aachen}

[1] Bemelmans J.: Die Vorlesung "Figur und Rotation der Himmelskörper" von F. Hausdorff, WS 1895/96, Universität Leipzig, S 20, März 2005

[2] Wagner A.: Optimal Shape Problems for Eigenvalues, S 30, März 2005

[3] Hildebrandt S. and von der Mosel H.: Conformal representation of surfaces, and Plateau's problem for Cartan functionals, S 43, Juli 2005

[4] Reiter P.: All curves in a $C^{1}$-neighbourhood of a given embedded curve are isotopic, S 8, Oktober 2005

[5] Maier-Paape S., Mischaikow K. and Wanner T.: Structure of the Attractor of the Cahn-Hilliard Equation, S 68, Oktober 2005

[6] Strzelecki P. and von der Mosel H.: On rectifiable curves with $L^{p}$ bounds on global curvature: Self-avoidance, regularity, and minimizing knots, S 35, Dezember 2005

[7] Bandle C. and Wagner A.: Optimization problems for weighted Sobolev constants, S 23, Dezember 2005

[8] Bandle C. and Wagner A.: Sobolev Constants in Disconnected Domains, S 9, Januar 2006

[9] McKenna P.J. and Reichel W.: A priori bounds for semilinear equations and a new class of critical exponents for Lipschitz domains, S 25, Mai 2006

[10] Bandle C., Below J. v. and Reichel W.: Positivity and anti-maximum principles for elliptic operators with mixed boundary conditions, S 32, Mai 2006

[11] Kyed M.: Travelling Wave Solutions of the Heat Equation in Three Dimensional Cylinders with Non-Linear Dissipation on the Boundary, S 24, Juli 2006

[12] Blatt S. and Reiter P.: Does Finite Knot Energy Lead To Differentiability?, S 30, September 2006

[13] Grunau H.-C., Ould Ahmedou M. and Reichel W.: The Paneitz equation in hyperbolic space, S 22, September 2006

[14] Maier-Paape S., Miller U.,Mischaikow K. and Wanner T.: Rigorous Numerics for the Cahn-Hilliard Equation on the Unit Square, S 67, Oktober 2006

[15] von der Mosel H. and Winklmann S.: On weakly harmonic maps from Finsler to Riemannian manifolds, S 43, November 2006

[16] Hildebrandt S., Maddocks J. H. and von der Mosel H.: Obstacle problems for elastic rods, S 21, Januar 2007

[17] Galdi P. Giovanni: Some Mathematical Properties of the Steady-State Navier-Stokes Problem Past a ThreeDimensional Obstacle, S 86, Mai 2007

[18] Winter N.: $W^{2, p}$ and $W^{1, p}$-estimates at the boundary for solutions of fully nonlinear, uniformly elliptic equations, S 34, July 2007

[19] Strzelecki P., Szumańska M. and von der Mosel H.: A geometric curvature double integral of Menger type for space curves, S 20, September 2007

[20] Bandle C. and Wagner A.: Optimization problems for an energy functional with mass constraint revisited, S 20, März 2008

[21] Reiter P., Felix D., von der Mosel H. and Alt W.: Energetics and dynamics of global integrals modeling interaction between stiff filaments, S 38, April 2008

[22] Belloni M. and Wagner A.: The $\infty$ Eigenvalue Problem from a Variational Point of View, S 18, Mai 2008

[23] Galdi P. Giovanni and Kyed M.: Steady Flow of a Navier-Stokes Liquid Past an Elastic Body, S 28, Mai 2008

[24] Hildebrandt S. and von der Mosel H.: Conformal mapping of multiply connected Riemann domains by a variational approach, S 50, Juli 2008

[25] Blatt S.: On the Blow-Up Limit for the Radially Symmetric Willmore Flow, S 23, Juli 2008 
[26] Müller F. and Schikorra A.: Boundary regularity via Uhlenbeck-Rivière decomposition, S 20, Juli 2008

[27] Blatt S.: A Lower Bound for the Gromov Distortion of Knotted Submanifolds, S 26, August 2008

[28] Blatt S.: Chord-Arc Constants for Submanifolds of Arbitrary Codimension, S 35, November 2008

[29] Strzelecki P., Szumańska M. and von der Mosel H.: Regularizing and self-avoidance effects of integral Menger curvature, S 33, November 2008

[30] Gerlach H. and von der Mosel H.: Yin-Yang-Kurven lösen ein Packungsproblem, S 4, Dezember 2008

[31] Buttazzo G. and Wagner A.: On some Rescaled Shape Optimization Problems, S 17, März 2009

[32] Gerlach H. and von der Mosel H.: What are the longest ropes on the unit sphere?, S 50, März 2009

[33] Schikorra A.: A Remark on Gauge Transformations and the Moving Frame Method, S 17, Juni 2009

[34] Blatt S.: Note on Continuously Differentiable Isotopies, S 18, August 2009

[35] Knappmann K.: Die zweite Gebietsvariation für die gebeulte Platte, S 29, Oktober 2009

[36] Strzelecki P. and von der Mosel H.: Integral Menger curvature for surfaces, S 64, November 2009

[37] Maier-Paape S., Imkeller P.: Investor Psychology Models, S 30, November 2009

[38] Scholtes S.: Elastic Catenoids, S 23, Dezember 2009

[39] Bemelmans J., Galdi G.P. and Kyed M.: On the Steady Motion of an Elastic Body Moving Freely in a Navier-Stokes Liquid under the Action of a Constant Body Force, S 67, Dezember 2009

[40] Galdi G.P. and Kyed M.: Steady-State Navier-Stokes Flows Past a Rotating Body: Leray Solutions are Physically Reasonable, S 25, Dezember 2009

[41] Galdi G.P. and Kyed M.: Steady-State Navier-Stokes Flows Around a Rotating Body: Leray Solutions are Physically Reasonable, S 15, Dezember 2009

[42] Bemelmans J., Galdi G.P. and Kyed M.: Fluid Flows Around Floating Bodies, I: The Hydrostatic Case, S 19, Dezember 2009

[43] Schikorra A.: Regularity of n/2-harmonic maps into spheres, S 91, März 2010

[44] Gerlach H. and von der Mosel H.: On sphere-filling ropes, S 15, März 2010

[45] Strzelecki P. and von der Mosel H.: Tangent-point self-avoidance energies for curves, S 23, Juni 2010

[46] Schikorra A.: Regularity of n/2-harmonic maps into spheres (short), S 36, Juni 2010

[47] Schikorra A.: A Note on Regularity for the $n$-dimensional H-System assuming logarithmic higher Integrability, S 30, Dezember 2010

[48] Bemelmans J.: Über die Integration der Parabel, die Entdeckung der Kegelschnitte und die Parabel als literarische Figur, S 14, Januar 2011

[49] Strzelecki P. and von der Mosel H.: Tangent-point repulsive potentials for a class of non-smooth $m$-dimensional sets in $\mathbb{R}^{n}$. Part I: Smoothing and self-avoidance effects, S 47, Februar 2011

[50] Scholtes S.: For which positive $p$ is the integral Menger curvature $\mathcal{M}_{p}$ finite for all simple polygons, S 9, November 2011

[51] Bemelmans J., Galdi G. P. and Kyed M.: Fluid Flows Around Rigid Bodies, I: The Hydrostatic Case, S 32, Dezember 2011

[52] Scholtes S.: Tangency properties of sets with finite geometric curvature energies, S 39, February 2012

[53] Scholtes S.: A characterisation of inner product spaces by the maximal circumradius of spheres, S 8, February 2012 